\title{
Consumo de pipas de agua y cigarrillos entre adolescentes de Sevilla (España): prevalencia y potenciales determinantes
}

\section{Waterpipe and cigarette smoking among adolescents in Seville (Spain): prevalence and potential determinants}

\author{
Juan Manuel Sáenz-Lussagnet*, Fernando Rico-Villademoros**, Luis Gabriel LuQue-Romero***. \\ * Técnico de Promoción de la Salud. Distrito Sanitario Aljarafe-Sevilla Norte, Sevilla. ** Instituto de Neurociencias. Universidad \\ de Granada, Granada. *** Unidad de Investigación. Distrito Sanitario Aljarafe-Sevilla Norte, Sevilla.
}

$\mathrm{E}$ 1 consumo de tabaco en pipas de agua se asocia a importantes problemas de salud (Waziry, Jawad, Ballout, Al Akel, y Akl, 2017) y, dado su crecimiento en los últimos años, se está convirtiendo en un problema de salud pública a nivel mundial, en especial entre los jóvenes, representando una nueva amenaza en la lucha global contra el tabaco y sus consecuencias en términos de morbilidad y mortalidad (Maziak et al., 2017; WHO Study Group on Tobacco Product Regulation (TobReg), 2015).

En España, los datos sobre el consumo de pipas de agua son muy limitados (Agaku et al., 2014; Jorge-Araujo, Torres-García, Saavedra-Santana, y Navarro-Rodríguez, 2017). Como parte de un proyecto sobre prevención del consumo de tabaco en adolescentes se realizó este estudio transversal entre abril y mayo de 2014 en tres institutos de enseñanza secundaria (IES) de Sevilla (3057 alumnos), cuyos objetivos fueron evaluar la prevalencia de consumo de pipas de agua y cigarrillos entre adolescentes y analizar los posibles determinantes y creencias en torno al consumo de pipas de agua. Para ello, se aplicó de forma anónima un cuestionario autoadministrado ad-hoc de 12 preguntas sobre consumo de tabaco y creencias (disponible bajo solicitud) creado de forma consistente con las recomendaciones de los expertos (Maziak, Ward, Afifi Soweid, y Eissenberg, 2005).

En cada IES, se seleccionó, mediante una tabla de números aleatorios, una clase por cada curso. De los 501 participantes de las clases seleccionadas, 139 (28\%, intervalo de confianza [IC] al 95\% 24 a 32) presentaban un consumo de tabaco (cigarrillos o pipas de agua) consolidado (ver definiciones de consumo a pie de tabla). Con independencia del consumo concomitante de la otra modalidad, 66 (13\%, IC95\% 10 a 16) estudiantes presentaban consumo consolidado de pipas de agua, y 93 (19\%, IC95\% 15 a 22) consumo consolidado de cigarrillos; del mismo modo, 343 (69\%, IC95\% 64 a 73) estudiantes habían consumido alguna vez pipas de agua y 253 (51\%, IC95\%, 46 a 55) habían consumido alguna vez cigarrillos.

El análisis bivariante de los potenciales determinantes sociodemográficos del consumo de pipas de agua se presentan en la tabla. A destacar que en aquellos que nunca habían consumido pipas de agua, el $56 \%$ convivían con algún familiar fumador habitual, mientras que el $80 \%$ de los estudiantes con consumo consolidado convivían con fumadores habituales; según aumentaba el número de convivientes fumadores aumentaba la prevalencia de consumo consolidado de pipas de agua y de cigarrillos entre los estudiantes (Figura). Los estudiantes con consumo consolidado de pipas de agua tenían con más frecuencia un consumo consolidado de cigarrillos (un 30\%), mientras que en aquellos que nunca habían consumido pipas de agua también era infrecuente el consumo consolidado de cigarrillos (un 4\%).

Entre los consumidores consolidados de pipas agua era más frecuente que consideraran que este tipo de consumo es menos perjudicial para la salud que los cigarrillos,

Recibido: Enero 2018; Aceptado: Julio 2018.

Enviar correspondencia a:

Luis Gabriel Luque-Romero. Calle Echegaray, $n^{\circ} 1-1^{\circ}$ D. 41005-Sevilla (España).

Correo electrónico: luqueluis2@gmail.com; luisg.luque.sspa@juntadeandalucia.es. Teléfono móvil: 650912648 
que no afecta a los fumadores pasivos, que los paquetes de tabaco para pipas de agua detallan toda la información y avisos sobre los compuestos y aditivos que contiene, que no produce dependencia, y que no induce el consumo de cigarrillos $(p<0.001)$. Casi dos terceras partes de los estudiantes (una tercera parte entre aquellos con consumo consolidado de pipas de agua) desconocían si los paquetes de tabaco para pipas de agua especifican toda la información y avisos sobre los compuestos y aditivos que contiene.

En conclusión, nuestro estudio pone de manifiesto una elevada prevalencia de consumo consolidado de pipas de agua entre adolescentes (13\%), que, de confirmarse a nivel nacional, estaría entre las más altas comunicadas en distintos países o áreas geográficas (Agaku et al., 2014; Akl et al., 2011), a nivel de la población árabe de Estados Unidos (12-15\%) o la del Golfo Pérsico (9-16\%) (Akl et al., 2011). Este consumo de pipas de agua contribuye de forma rele- vante al consumo total de tabaco entre adolescentes $(28 \%$ en nuestra encuesta), con cifras superiores en nuestro estudio a las comunicadas sobre consumo de tabaco entre adolescentes para España en la encuesta Health Behaviour in School-aged Children de 2014 (Moreno et al., 2016); esta última encuesta incluirá en próximas ediciones el consumo de pipas de agua y otras modalidades de consumo de tabaco, proporcionando datos más ajustados de consumo actual de éste (Moreno, Ramos, y Rivera, 2017). Aunque limitado por el diseño transversal, en consonancia con otros estudios (Jiang, Ho, Wang, Leung, y Lam, 2017), nuestros datos sugieren que el consumo de pipas de agua se asocia al consumo de cigarrillos. Además de la necesidad de estudios a nivel nacional, estos resultados sugieren que son necesarias la adopción de medidas preventivas que deben iniciarse de forma precoz -antes de $3^{\circ}$ de la ESO- (Diaz Geada, Busto Miramontes, y Caamano Isorna, 2018) diri-

Tabla 1. Análisis bivariante de la relación entre las características demográficas y el consumo de pipas de agua.

\begin{tabular}{|c|c|c|c|c|}
\hline \multirow[b]{3}{*}{ Característica } & \multicolumn{3}{|c|}{ Consumo de pipas de agua } & \multirow{3}{*}{ valor de $p$} \\
\hline & Nunca & No consolidado* & Consolidado* & \\
\hline & $N=158$ & $N=277$ & $N=66$ & \\
\hline Edad (años), P50 (P25-P75) & $15.5(14.0-17.4)$ & $17.4(15.5-18.4)$ & $16.5(15.3-17.5)$ & $<0.001^{\ddagger}$ \\
\hline Sexo, N (\%) & & & & $0,18^{\#}$ \\
\hline Hombre & $78(49.4)$ & $133(48.0)$ & $40(60.6)$ & \\
\hline Mujer & $80(50.6)$ & $144(52.0)$ & $26(39.4)$ & \\
\hline Tipo de centro, N (\%) & & & & $<0.001^{\#}$ \\
\hline Rural $(\mathrm{IH})$ & $39(24.7)$ & $59(21.3)$ & $31(47.0)$ & \\
\hline Periurbano $(\mathrm{CL})$ & $62(39.2)$ & $128(46.2)$ & $25(37.9)$ & \\
\hline Metropolitano (JM) & $57(36.1)$ & $90(32.5)$ & $10(15.2)$ & \\
\hline Nivel de estudio, N (\%) & & & & $<0.001^{\#}$ \\
\hline $1 \stackrel{0}{\mathrm{ESO}}$ & $37(25.0)$ & $25(10.2)$ & $8(12.7)$ & \\
\hline $2^{\circ} \mathrm{ESO}$ & $29(19.6)$ & $19(7.8)$ & $7(11.1)$ & \\
\hline $3^{\circ} \mathrm{ESO}$ & $24(16.2)$ & $42(17.1)$ & $15(23.8)$ & \\
\hline 4- $\mathrm{ESO}$ & $16(10.8)$ & $39(15.9)$ & $14(22.2)$ & \\
\hline 1ํㅡ Bachiller & $30(20.3)$ & $69(28.2)$ & $13(20.6)$ & \\
\hline $2^{\circ}$ Bachiller & $12(8.1)$ & $51(20.8)$ & $6(9.5)$ & \\
\hline Módulo & $10(6.3)$ & $32(11.6)$ & $3(4.5)$ & \\
\hline Familiar fumador habitual, N (\%) & & & & $0,001^{\#}$ \\
\hline No & $70(44.3)$ & $86(31.0)$ & $13(19.7)$ & \\
\hline $\mathrm{Si}$ & $88(55.7)$ & $191(69.0)$ & $53(80.3)$ & \\
\hline Consumo de cigarrillos ${ }^{\dagger}, \mathrm{N}(\%)$ & & & & $<0.001^{\#}$ \\
\hline Consolidado & $6(3.8)$ & $67(24.2)$ & $20(30.3)$ & \\
\hline
\end{tabular}

Nota. ${ }^{*}$ Consumo consolidado de pipas de agua si el consumo era semanal o diario; consumo no consolidado si el consumo era mensual o esporádico. $\dagger$ Consumo consolidado de cigarrillos si el consumo era semanal o diario; consumo no consolidado si consumía esporádicamente (“alguna vez”).

CL, Instituto de Enseñanza Secundaria Carmen Laffón de San José de la Rinconada; ESO, Enseñanza Secundaria Obligatoria; IH, Instituto de Enseñanza Secundaria Heliche de Olivares; JM, Instituto de Enseñanza Secundaria Juan de Mairena de Mairena del Aljarafe; P, percentil.

Contaste de hipótesis bilateral, nivel de confianza 95\%; ${ }^{*}$ test U Mann-Whitney; ${ }^{~}$ ji-cuadrado de Pearson (IBM-SPSS v.18). 


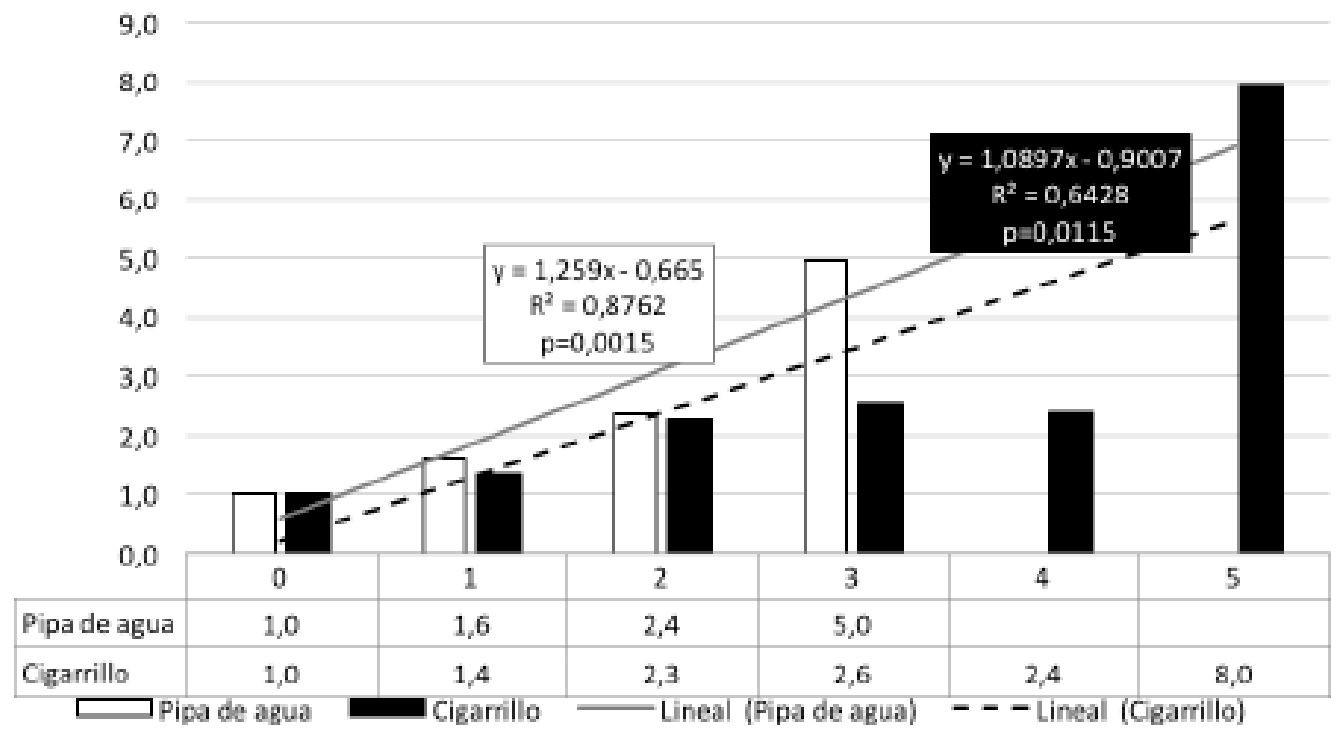

Figura 1. Razones de prevalencia de consumo consolidado de cigarrillos y pipas de agua dependiendo del número de familiares convivientes fumadores habituales.

Nota. Las razones de prevalencia (eje de ordenadas) se han calculado para cada categoría de número de familiares convivientes fumadores (eje de abscisas) como el cociente entre la prevalencia de consumo consolidado en dicha categoría y la prevalencia en la categoría de '0' familiares convivientes fumadores (EPIDAT 3.1).

gidas a desmitificar el carácter menos perjudicial de este consumo y el de cigarrillo electrónico (González Roz, Secades Villa, y Weidberg, 2017) entre los niños y adolescentes, medidas que deben alcanzar el medio familiar y el desarrollo de la normativa específica que regula dicho consumo.

\section{Reconocimientos}

A los institutos de Enseñanza Secundarias dependientes de la Delegación Territorial de Educación de la Provincia de Sevilla: Carmen Laffón (San José de la Rinconada), Heliche (Olivares) y Juan de Mairena (Mairena del Aljarafe). $\mathrm{A} \mathrm{D}^{\mathrm{a}}$. Noelia Adame Romero, Técnica del Área de Juventud del Ayuntamiento de San José de la Rinconada.

\section{Conflicto de intereses}

En nombre de todos los autores, el primer firmante del manuscrito declara que no existe ningún conflicto de intereses en relación con este artículo.

\section{Referencias}

Agaku, I. T., Filippidis, F. T., Vardavas, C. I., Odukoya, O. O., Awopegba, A. J., Ayo-Yusuf, O. A. y Connolly, G. N. (2014). Poly-tobacco use among adults in 44 countries during 2008-2012: evidence for an integrative and comprehensive approach in tobacco control. Drug and Alcohol Dependence, 139, 60-70. doi:10.1016/j.drugalcdep.2014.03.003.
Akl, E. A., Gunukula, S. K., Aleem, S., Obeid, R., Jaoude, P. A., Honeine, R. y Irani, J. (2011). The prevalence of waterpipe tobacco smoking among the general and specific populations: a systematic review. BMC Public Health, 11, 244. doi:10.1186/1471-2458-11-244.

Diaz Geada, A., Busto Miramontes, A. y Caamano Isorna, F. (2018). Alcohol, tobacco and cannabis consumption in adolescents from a multicultural population (Burela, Lugo). Adicciones, 30, 264-270. doi: 10.20882/adicciones.915.

González Roz, A., Secades Villa, R. y Weidberg, S. (2017). Evaluating nicotine dependence levels in e-cigarette users. Adicciones, 29, 136-138. doi:10.20882/adicciones.905.

Jiang, N., Ho, S. Y., Wang, M. P., Leung, L. T. y Lam, T. H. (2017). The relationship of waterpipe use with cigarette smoking susceptibility and nicotine dependence: A cross-sectional study among Hong Kong adolescents. Addictive Behaviors, 64, 123-128. doi:10.1016/j.addbeh.2016.08.035.

Jorge-Araujo, P., Torres-García, M., Saavedra-Santana, P. y Navarro-Rodríguez, C. ( 2017). Consumo de tabaco en pipa de agua en estudiantes españoles de secundaria y bachillerato de la provincia de Las Palmas. Salud y Drogas, 17, 121-131.

Maziak, W., Ben Taleb, Z., Jawad, M., Afifi, R., Nakkash, R., Akl, E. A., ... Eissenberg, T. (2017). Consensus statement on assessment of waterpipe smoking in epidemiological studies. Tobacco Control, 26, 338-343. doi:10.1136/tobaccocontrol-2016-052958. 
Maziak, W., Ward, K. D., Afifi Soweid, R. A. y Eissenberg, T. (2005). Standardizing questionnaire items for the assessment of waterpipe tobacco use in epidemiological studies. Public Health, 119, 400-404. doi:10.1016/j. puhe.2004.08.002.

Moreno, C., Ramos, P. y Rivera, F. (2017). Informe descriptivo de la propuesta de cuestionario a utilizar por el Health Behaviour in School-aged Children (HBSC) en su edición de 2018. Manuscrito remitido al Ministerio de Sanidad, Servicios Sociales e Igualdad.

Moreno, C., Ramos, P., Rivera, F., Jiménez-Iglesias, A., García-Moya, I., Sánchez-Queija, I. y Morgan, A. (2016). Informe técnico de los resultados obtenidos por el estudio Health Behaviour in School-aged Children (HBSC) 2014 en España. Recuperado de https://www.msssi.gob.es/profesionales/saludPublica/prevPromocion/promocion/saludJovenes/estudioHBSC/HBSC2014_InformeTecnico.htm.

Waziry, R., Jawad, M., Ballout, R. A., Al Akel, M. y Akl, E. A. (2017). The effects of waterpipe tobacco smoking on health outcomes: an updated systematic review and meta-analysis. International Journal of Epidemiology, 46, 32-43. doi:10.1093/ije/dyw021.

WHO Study Group on Tobacco Product Regulation (TobReg) (2015). Advisory note: waterpipe tobacco smoking: health effects, research needs and recommended actions by regulators Recuperado de http://apps.who.int/iris/ bitstream/10665/161991/1/9789241508469_eng.pdf?ua $=1 \& u a=1$. 